\title{
THE STRUCTURE OF LOCAL INTEGRAL ORTHOGONAL GROUPS
}

\author{
BY
}

D. G. JAMES( $\left.{ }^{1}\right)$

\begin{abstract}
Let $M$ be a lattice on a regular quadratic space over a nondyadic local field. The normal subgroups of the integral orthogonal group $O(M)$ are determined.
\end{abstract}

1. Introduction. Let $F$ be a field complete with respect to a discrete valuation, $\mathfrak{o}$ its ring of integers and $\mathfrak{p}$ the unique maximal ideal in $\mathfrak{o}$. Assume that 2 is a unit in $\mathrm{o}$. Let $V$ be a finite dimensional nonsingular quadratic space over $F$ and $M$ an o-lattice on $V$. The structure of the orthogonal groups $O(V)$ and $O(M)$ have been studied by many authors. By structure, we mean here primarily a classification of the normal subgroups.

In particular, Klingenberg [5] considered the case where $M$ is an isotropic unimodular lattice with rank at least three (and o a local ring) and obtained a classification in terms of congruence subgroups. Riehm [10] generalized this to lattices $M$ where each modular Jordan component is isotropic with rank at least three, introducing the concept of a tableau, a matrix of ideals, to define the more general congruence subgroups necessary for the classification. This local information is also relevant to the study of orthogonal groups of forms over rings of algebraic integers.

In another direction, if $V$ is anisotropic and $M$ is a maximal lattice on $V$, then $O(V)=O(M)$. If $F$ is now a local field (and the dimension of $V$ is 3 or 4), Pollak [8] and Johnson [4] classified the normal subgroups of the commutator subgroup of $O(V)$ by means of conguence subgroups, utilizing results on quaternion algebras. Later, Pollak [9] and Martin [6] studied the normal subgroups in $O^{+}(V)$ when $F=\mathbf{R}((X))$ is the field of formal power series over the real field $\mathbf{R}$, using a combination of the above techniques. Again, Bröcker [1] studied $O(V)$ over arbitrary complete discrete valuation rings by means of a filter on the topological group $O(V)$. In this connection one should note that the noncentral normal subgroups are open in the p-adic topology on $O(V)$.

Received by the editors August 25, 1975.

AMS (MOS) subject classifications (1970). Primary 20G25, $10 \mathrm{C} 30$.

(1) This research was partially supported by the National Science Foundation. 
The main aim in the present paper is to extend Riehm's classification by dropping the restrictions on the modular Jordan components of $M$. At the same time, the method employed here is applicable to anisotropic spaces and connections with the work of Pollak et al.will be indicated at the appropriate points. Some of the ideas used here have already appeared in an earlier work of the author [3].

In the first part of this paper we introduce two families of congruence subgroups $O(\mathfrak{f})$ and $E(\mathfrak{f})$, defined with the help of a family of ideals $\mathfrak{f}=\left\{\mathfrak{a}_{i j}\right\}$ satisfying certain restrictions determined by the scales of the Jordan components of $M$. The family $f$ is similar, but more general, to the tableau used by Riehm. Generators for $O(\mathfrak{f})$ and $E(\mathfrak{f})$ are given, and the quotient group $O(\mathfrak{f}) / E(\mathfrak{f})$ determined. The results at this point are for arbitrary residue class fields.

Then, assuming that the residue class field is finite, it is shown that any open normal subgroup $\mathfrak{N}$ of $O(M)$ satisfies

$$
E(\mathfrak{f}) \subseteq \mathfrak{N} \cong I(\mathfrak{f}) O(\mathfrak{f})
$$

for a suitable unique family of ideals $\mathfrak{f}$ (provided, if $F$ is not a completion of the field of rational numbers, at most one Jordan component of $M$ has rank one, for otherwise exceptional behavior occurs). Here $I(\mathfrak{f})$ is a finite abelian group of exponent two; moreover, for all but a finite number of $f, I(f)$ is the center of $O(M)$. This reduces the classification of normal subgroups to the study of the quotient groups $O(\mathfrak{f}) / E(\mathfrak{f})$. These groups are direct products of quotient congruence groups defined on the modular Jordan components of $M$. Thus the classification has been reduced to the modular case and so depends primarily on the ranks of the Jordan components of $M$ (in particular, whether this rank is 1,2 or $\geqslant 3$ ). In special cases very tight results are available. An example illustrating the exceptional behavior mentioned above is also given.

Our notation and terminology follows that in O'Meara [7] and, aside from this reference, the presentation here is essentially self-contained.

2. Congruence subgroups. Let $\mathfrak{o}$ be the ring of integers in a field $F$ complete with respect to a discrete valuation. The unique maximal ideal in $\mathcal{o}$ will be denoted by $\mathfrak{p}$ and we assume that the characteristic of the residue class field $\mathfrak{o} / \mathfrak{p}$ is not two; further restrictions on this residue class field will be imposed later. Let $V$ be a regular finite dimensional quadratic space over $F$ and $M$ a lattice on $V$ with a Jordan decomposition

$$
M=M_{1} \perp M_{2} \perp \cdots \perp M_{h}
$$

where each $M_{i}$ is a modular lattice with scale $\unlhd M_{i}=\xi_{i}=\mathfrak{p}^{e_{i}}, 1 \leqslant i \leqslant h$, and the $M_{i}$ are ordered so that $\mathfrak{g}_{1} \supset \mathfrak{g}_{2} \supset \ldots \supset \mathfrak{g}_{h}$. It is assumed, henceforth, that 
$h \geqslant 2$, although much of the following allows $h$ to be one.

If $q: V \rightarrow F$ denotes the quadratic form on $V$, the orthogonal group of $V$ is

$$
O(V)=\{\varphi \in \mathrm{GL}(V) \mid q(\varphi(x))=q(x) \text { for all } x \in V\}
$$

and the orthogonal group of $M$ is

$$
O(M)=\{\varphi \in O(V) \mid \varphi(M)=M\} .
$$

Both the groups $O(V)$ and $O(M)$ are topological groups inheriting a $\mathfrak{p}$-adic topology from the topology on $F$. Our object is to classify the (open) normal subgroups in $O(M)$.

Let $C(V)$ be the Clifford algebra of the quadratic space $V$ obtained by factoring out in the tensor algebra of $V$ the ideal generated by elements of the form $r \otimes r-q(r) 1$ with $r$ in $V$. Identify $V$ with the natural isomorphic image in $C(V)$ and use $\otimes$ for the multiplication in the Clifford algebra. Then

$$
r \otimes s+s \otimes r=B(r, s) 1
$$

where

$$
B(r, s)=q(r+s)-q(r)-q(s)
$$

is the symmetric bilinear form associated with $q$. We will find it very helpful to view many of our isometries as inner automorphisms of the Clifford algebra. In particular, relations between isometries are often most easily established by a suitable multiplication in the algebra.

Take $r$ and $s$ in $V$ with $B(r, s)=0$. Then

$$
(1+r \otimes s) \otimes(1+s \otimes r)=1+q(r) q(s) .
$$

If $1+q(r) q(s)$ is not zero, we define an isometry $E(r, s)$ on $V$ by the inner automorphism

$$
\begin{aligned}
E(r, s)(x) & =(1+r \otimes s) \otimes x \otimes(1+r \otimes s)^{-1} \\
& =x+(1+q(r) q(s))^{-1}(B(x, s-q(s) r) r-B(x, r+q(r) s) s)
\end{aligned}
$$

for $x$ in $V$. If $q(r)=0, t$, ; isometry reduces to the Eichler-Siegel transformation

$$
E(r, s)(x)=x-B(x, r) s+B(x, s) r-q(s) B(x, r) r .
$$

The isometry $E(r, s)$ has properties sufficiently similar to the Eichler-Siegel transformation for us to duplicate many arguments from the isotropic situation. Thus, it is easily seen for any $\theta$ in $O(V)$, 


$$
\theta E(r, s) \theta^{-1}=E(\theta(r), \theta(s))
$$

Also,

$$
E(r, s)^{-1}=E(r,-s)=E(s, r)
$$

and, for $\alpha$ in $F$,

$$
E(\alpha r, s)=E(r, \alpha s)
$$

Denote by $O\left(M_{i}\right), 1 \leqslant i \leqslant h$, the subgroup of $O(M)$ consisting of those isometries which act identically on the orthogonal complement $M_{i}^{\perp}$ of $M_{i}$ in $M$. For an ideal $a$ in $\mathrm{o}$, define $O\left(\mathrm{a} M_{i}\right)$ to be the subgroup of those $\varphi$ in $O\left(M_{i}\right)$ for which

$$
\varphi(x) \equiv x \bmod a M_{i}
$$

for all $x$ in $M_{i}$.

Let ord denote a normalized valuation on $F$. Since the characteristic of $0 / p$ is not two, each modular component $M_{i}, 1 \leqslant i \leqslant h$, has an integral orthogonal basis.

LEMMA 2.1. Fix $i$ and let $x_{1}, \ldots, x_{n}$ be an orthogonal basis for $M_{i}$. If $n=\operatorname{rank} M_{i} \geqslant 2$ and $\mathfrak{a}$ is an ideal in $\mathfrak{p}$, then the group $O\left(a M_{j}\right)$ is generated by isometries of the form $E\left(x_{j}, y_{j}\right)$ where each $y_{j}$ is in $a_{s_{i}}^{-1} M_{i}$ and $B\left(x_{j}, y_{k}\right)$ $=0$ for $1 \leqslant j \leqslant k \leqslant n-1$.

Proof. Take $\varphi$ in $O\left(a M_{i}\right)$ and let

$$
\varphi\left(x_{1}\right)=\alpha x_{1}+y \equiv x_{1} \bmod a M_{i}
$$

where $B\left(x_{1}, y\right)=0$. Then $\alpha \equiv 1 \bmod a$ and $y \in a M_{i}$. Choose $\beta$ in $F$ such that $\alpha+\beta q(y)=1$. Since $q\left(\varphi\left(x_{1}\right)\right)=q\left(x_{1}\right)$, it follows that $\beta(1+\alpha) q\left(x_{1}\right)$ $=1$, so that ord $\beta=-e_{i}$ and $\beta y \in a \xi_{i}^{-1} M_{i}$. A short calculation shows that $E\left(x_{1}, \beta y\right) \varphi\left(x_{1}\right)=x_{1}$. Note also that $E\left(x_{1}, \beta y\right)$ is in $O\left(\mathfrak{a} M_{i}\right)$. The proof can now be completed by induction on the rank of $M_{i}$.

For any fractional ideal $r$, define

$$
M(\mathfrak{r})=\{x \in M \mid B(x, M) \subseteq \mathfrak{r}\} .
$$

$M(\mathrm{r})$ is a lattice on $V$, invariant as a set under the action of $O(M)$. Moreover, if $r_{1} \supseteqq r_{2}$, then

$$
M\left(\mathrm{r}_{1}\right) \supseteqq M\left(\mathrm{r}_{2}\right)
$$

and

$$
\mathrm{r}_{1}^{-1} M\left(\mathrm{r}_{1}\right) \subseteq \mathrm{r}_{2}^{-1} M\left(\mathrm{r}_{2}\right) .
$$

In particular, for $1 \leqslant i \leqslant h$, we have 


$$
M\left(\mathfrak{s}_{i}\right)=\left(\mathfrak{s}_{i}: \mathfrak{s}_{1}\right) M_{1} \perp \cdots \perp\left(\mathfrak{s}_{i}: \mathfrak{s}_{i-1}\right) M_{i-1} \perp M_{i} \perp \cdots \perp M_{h}
$$

where the $\mathfrak{s}_{i}$ are the scales of the $M_{i}$ and (a: $\left.\mathfrak{b}\right)$ denotes the quotient fractional ideal $\mathfrak{a b}^{-1}$. In comparing our results with Riehm [10], however, one should note that he takes $(\mathfrak{a}: \mathfrak{b})$ to be the quotient integral ideal in the ring theoretic sense, so that $(\mathfrak{a}: \mathfrak{b})=\mathfrak{o}$ whenever $\mathfrak{b} \subseteq \mathfrak{a}$.

We also need the dual lattice $M^{\sharp}$ of $M$. Here

$$
M^{\sharp}=\{x \in V \mid B(x, M) \subseteq 0\}=s_{1}^{-1} M_{1} \perp \cdots \perp s_{h}^{-1} M_{h} .
$$

Moreover, for $1 \leqslant i \leqslant h$,

$$
\mathfrak{\Xi}_{i}^{-1} M\left(\mathfrak{\xi}_{i}\right) \cong M^{\sharp} .
$$

For any ideal $\mathfrak{a} \subseteq \mathfrak{p}$ and for $1 \leqslant i \leqslant j \leqslant h$, define $E_{i j}(\mathfrak{a})$ to be the subgroup of $O(M)$ generated by isometries of the form $E(r, s)$ where $r$ is in $\mathfrak{a} \mathfrak{S}_{i}^{-1} M\left(\mathfrak{s}_{i}\right), s$ is in $M\left(\mathfrak{S}_{j}\right)$ and $B(r, s)=0$. If $i \neq j$, then $\mathfrak{a}=\mathfrak{o}$ is also allowed in the definition. The $E_{i j}(a)$ are clearly normal subgroups in $O(M)$. Note that such an $E(r, s)$ is an isometry in $O(M)$ since $1+q(r) q(s)$ is a unit,

$$
B(M, s-q(s) r) r \cong \mathfrak{a}_{j} \mathfrak{g}_{i}^{-1} M\left(\mathfrak{s}_{i}\right) \leqq \mathfrak{a} M\left(\mathfrak{g}_{j}\right)
$$

and

$$
B(M, r+q(r) s) s \leqq a M\left(\mathfrak{\Im}_{j}\right) .
$$

LEMMA 2.2. For $1 \leqslant i \leqslant j \leqslant k \leqslant h$,

$$
E_{j k}\left(\left(\mathfrak{j}_{j}: \mathfrak{s}_{i}\right) \mathfrak{a}\right) \cong E_{i k}(\mathfrak{a}) \leqq E_{j k}(\mathfrak{a})
$$

and

$$
E_{i j}\left(\left(\mathfrak{g}_{k}: \mathfrak{s}_{j}\right) \mathfrak{a}\right) \cong E_{i k}(\mathfrak{a}) \cong E_{i j}(\mathfrak{a})
$$

for those ideals a where the groups are defined.

Proof. These inclusions follow from

$$
\left(\mathfrak{S}_{j}: \mathfrak{S}_{i}\right) \mathfrak{S}_{j}^{-1} M\left(\mathfrak{\mathfrak { j }}_{j}\right) \cong \mathfrak{s}_{i}^{-1} M\left(\mathfrak{g}_{i}\right) \cong \mathfrak{g}_{j}^{-1} M\left(\mathfrak{g}_{j}\right)
$$

and

$$
\left(\mathfrak{I}_{k}: \mathfrak{S}_{j}\right) M\left(\mathfrak{g}_{j}\right) \cong M\left(\mathfrak{\Im}_{k}\right) \cong M\left(\mathfrak{\Im}_{j}\right)
$$

respectively. 
Lemma 2.3. For $1 \leqslant i<j \leqslant h$ and any ideal $\mathfrak{a} \subseteq \mathfrak{0}$,

$$
O\left(\mathfrak{a}\left(\mathfrak{g}_{j}: \mathfrak{S}_{i}\right) M_{i}\right) \subseteq E_{i j}(\mathfrak{a}) \text { and } O\left(\mathfrak{a}\left(\mathfrak{g}_{j}: \mathfrak{g}_{i}\right) M_{j}\right) \subseteq E_{i j}(\mathfrak{a})
$$

Proof. These follow from

$$
O\left(a\left(\mathfrak{g}_{j}: \mathfrak{S}_{i}\right) M_{i}\right) \subseteq E_{i i}\left(\mathfrak{a}\left(\mathfrak{S}_{j}: \mathfrak{S}_{i}\right)\right) \subseteq E_{i j}(\mathrm{a})
$$

and

$$
O\left(\mathrm{a}\left(\mathfrak{S}_{j}: \mathfrak{S}_{i}\right) M_{j}\right) \subseteq E_{j j}\left(\mathrm{a}\left(\mathfrak{S}_{j}: \mathfrak{S}_{i}\right)\right) \subseteq E_{i j}(\mathfrak{a})
$$

using Lemmas 2.1 and 2.2.

Definitron. A semitableau $f=\left\{a_{i j}\right\}$ (for $M$ ) is a family of ideals $a_{i j}, 1 \leqslant i$ $<j \leqslant h$, in $\mathrm{o}$ that satisfy the relations

$$
\left(\mathfrak{g}_{j}: \mathfrak{S}_{i}\right) \mathfrak{a}_{i k} \subseteq a_{j k} \subseteq a_{i k}
$$

and

$$
\left(\mathfrak{g}_{k}: \mathfrak{g}_{j}\right) a_{i k} \subseteq a_{i j} \subseteq a_{i k}
$$

for $1 \leqslant i<j<k \leqslant h$.

If $\mathfrak{f}=\left\{a_{i j}\right\}$ is a semitableau, define

$$
E(\mathfrak{f})=\prod_{1 \leqslant i<j \leqslant h} E_{i j}\left(\mathfrak{a}_{i j}\right) .
$$

$E(\mathfrak{f})$ is clearly a normal subgroup of $O(M)$. The relations in the definition of a semitableau ensure that the subgroups implicitly present in $E(\mathfrak{f})$ by Lemma 2.2 , explicitly occur in the product defining $E(f)$. Conversely, for a product of subgroups $E_{i j}\left(\mathfrak{a}_{i j}\right)$ of this type with the ideals $a_{i j}$ chosen maximally, the family $\mathfrak{f}=\left\{a_{i j}\right\}$ is a semitableau.

ExAmple 2.4. Fix indices $g<k$ and an ideal $a$. Define

$$
a_{i j}= \begin{cases}a & \text { for } i \leqslant g<k \leqslant j \\ \left(\mathfrak{I}_{k}: \mathfrak{g}_{j}\right) \mathfrak{a} & \text { for } i \leqslant g \text { and } j \leqslant k \\ \left(\mathfrak{I}_{i}: \mathfrak{g}_{g}\right) \mathfrak{a} & \text { for } g \leqslant i \text { and } k \leqslant j \\ \left(\mathfrak{I}_{k} \mathfrak{g}_{i}: \mathfrak{g}_{j} \mathfrak{g}_{g}\right) \mathfrak{a} & \text { for } g<i<j<k\end{cases}
$$

Then $\mathfrak{f}=\left\{\mathfrak{a}_{i j}\right\}$ is a semitablsilu and since, from Lemma 2.2, $E_{i j}\left(a_{i j}\right) \subseteq E_{g k}(a)$ for $1 \leqslant i<j \leqslant h$, it follows $. . E(\mathfrak{f})=E_{g k}(\mathfrak{a})$. 
If $\mathfrak{f}=\left\{\mathfrak{a}_{i j}\right\}$ is a semitableau, define the congruence subgroup $O(\mathfrak{f})$ to be the set of all isometries $\varphi$ in $O(M)$ such that for all $1 \leqslant g<k \leqslant h$ and for all $x$ in $M\left(\mathfrak{a}_{k}\right)$,

$$
\varphi(x) \equiv x \bmod a_{g k}\left(\mathfrak{g}_{k}: \mathfrak{g}_{g}\right)\left(\mathfrak{g}_{g} M^{\sharp}+M\right) .
$$

Clearly, each $O(\mathfrak{f})$ is a normal subgroup of $O(M)$.

LEMMa 2.5. For any semitableau $\mathfrak{f}$,

$$
E(\mathfrak{f}) \subseteq O(\mathfrak{f}) .
$$

Proof. Let $\mathfrak{f}=\left\{a_{i j}\right\}$ and $\varphi=\prod_{i<j} \varphi_{i j}$ be an isometry in $E(\mathfrak{f})$ where $\varphi_{i j}$ is in $E_{i j}\left(a_{i j}\right)$. Here $\varphi_{i j}$ is a product of isometries of the form $E(r, s)$ with $r$ in $\mathfrak{a}_{i j} \mathfrak{s}_{i}^{-1} M\left(\mathfrak{s}_{i}\right)$ and $s$ in $M\left(\mathfrak{g}_{j}\right)$. For $x$ in $M\left(\mathfrak{s}_{k}\right)$, we have $B(x, s-q(s) r) r$ and $B(x, r+q(r) s) s$ are in

$$
B\left(M\left(\mathfrak{g}_{k}\right), M\left(\mathfrak{s}_{j}\right)\right) \mathfrak{a}_{i j} \mathfrak{g}_{i}^{-1} M\left(\mathfrak{s}_{i}\right) \text { and } B\left(M\left(\mathfrak{g}_{k}\right), M\left(\mathfrak{s}_{i}\right)\right) \mathfrak{a}_{i j} \mathfrak{g}_{i}^{-1} M\left(\mathfrak{g}_{j}\right),
$$

respectively. It follows that

$$
\varphi_{i j}(x) \equiv x \bmod N
$$

where

$$
N=\mathfrak{a}_{i j} \mathfrak{s}_{i}^{-1}\left(\left(\mathfrak{a}_{k} \cap \mathfrak{s}_{j}\right) M\left(\mathfrak{a}_{i}\right)+\left(\mathfrak{g}_{k} \cap \mathfrak{s}_{i}\right) M\left(\mathfrak{s}_{j}\right)\right)
$$

Obviously, $N$ depends on the choice of $i, j$ and $k$. Note that

$$
\mathfrak{g}_{g} M^{\sharp}+M=M_{1} \perp \cdots \perp M_{g} \perp\left(\mathfrak{g}_{g}: \mathfrak{g}_{g+1}\right) M_{g+1} \perp \cdots \perp\left(\mathfrak{g}_{g}: \mathfrak{g}_{h}\right) M_{h}
$$

is invariant under the action of $O(M)$. To complete the proof it is enough to verify that

$$
N \cong \mathfrak{a}_{g k}\left(\mathfrak{g}_{k}: \mathfrak{g}_{g}\right)\left(\mathfrak{g}_{g} M^{\sharp}+M\right) .
$$

Case 1. Assume that $i \leqslant g$ and $j \leqslant k$. Then

$$
\begin{aligned}
N & =\mathfrak{a}_{i j}\left(\mathfrak{g}_{k}: \mathfrak{I}_{i}\right) M\left(\mathfrak{I}_{i}\right) \\
& \cong \mathfrak{a}_{i j}\left(\mathfrak{g}_{k}: \mathfrak{I}_{i}\right)\left(\mathfrak{g}_{g} M^{\#}+M\right) \\
& \cong \mathfrak{a}_{g k}\left(\mathfrak{g}_{k}: \mathfrak{g}_{g}\right)\left(\mathfrak{g}_{g} M^{\#}+M\right),
\end{aligned}
$$

using the restrictions on the ideals $a_{i j}$.

Case 2. Assume that $g<i<j \leqslant k$. Then 


$$
\begin{aligned}
N & =\mathfrak{a}_{i j}\left(\mathfrak{I}_{k}: \mathfrak{I}_{i}\right) M\left(\mathfrak{I}_{i}\right) \\
& \subseteq \mathfrak{a}_{g k}\left(\mathfrak{I}_{k}: \mathfrak{I}_{g}\right) \mathfrak{I}_{g}\left(\mathfrak{I}_{i}^{-1} M\left(\mathfrak{I}_{i}\right)\right) \\
& \subseteq \mathfrak{a}_{g k}\left(\mathfrak{I}_{k}: \mathfrak{I}_{g}\right)\left(\mathfrak{I}_{g} M^{\sharp}+M\right) .
\end{aligned}
$$

Case 3. Assume that $i \leqslant g<k<j$. Then

$$
\begin{aligned}
N & =\mathfrak{a}_{i j} \mathfrak{g}_{i}^{-1}\left(\mathfrak{g}_{j} M\left(\mathfrak{g}_{i}\right)+\mathfrak{g}_{k} M\left(\mathfrak{g}_{j}\right)\right) \\
& \subseteq \mathfrak{a}_{i j}\left(\mathfrak{g}_{j}: \mathfrak{g}_{i}\right)\left(M+\mathfrak{g}_{k} M^{\sharp}\right) \\
& \subseteq a_{g k}\left(\mathfrak{g}_{k}: \mathfrak{g}_{g}\right)\left(\mathfrak{g}_{g} M^{\sharp}+M\right) .
\end{aligned}
$$

Case 4. Assume that $g<i$ and $k<j$. Then

$$
\begin{aligned}
N & =\mathfrak{a}_{i j} \mathfrak{g}_{i}^{-1}\left(\mathfrak{g}_{j} M\left(\mathfrak{g}_{i}\right)+\left(\mathfrak{g}_{k} \cap \mathfrak{I}_{i}\right) M\left(\mathfrak{g}_{j}\right)\right) \\
& \subseteq \mathfrak{a}_{i j} \mathfrak{g}_{j} M^{\sharp} \\
& \subseteq a_{g k}\left(\mathfrak{g}_{k}: \mathfrak{g}_{g}\right)\left(\mathfrak{g}_{g} M^{\sharp}+M\right) .
\end{aligned}
$$

Thus, in all cases, the congruences defining $O(f)$ are satisfied by all isometries in $E(\mathrm{f})$.

Definition. Let $\mathfrak{f}=\left\{a_{i j}\right\}$ be a semitableau. For $1 \leqslant k \leqslant h$, define

$$
\mathfrak{b}_{k}=a_{k-1 k} \cap \mathfrak{a}_{k k+1}
$$

and

$$
\mathfrak{c}_{k}=a_{k-1 k}\left(\mathfrak{g}_{k}: \mathfrak{g}_{k-1}\right)+a_{k k+1}\left(\mathfrak{g}_{k+1}: \mathfrak{g}_{k}\right),
$$

where we use the convention that any term involving the subscripts 0 or $h+1$ is omitted. Thus $\mathfrak{b}_{1}=a_{12}$ and $\mathfrak{b}_{h}=a_{h-1 h}$. From the restrictions on a semitableau, it follows that $\mathfrak{c}_{k} \subseteq \mathfrak{b}_{k}$.

Lemma 2.6. Let $\mathfrak{f}=\left\{a_{i j}\right\}$ be a semitableau. Then, for $1 \leqslant i \leqslant h$,

$$
O\left(\mathfrak{b}_{i} M_{i}\right) \subseteq O(\mathfrak{f})
$$

Proof. We must show that isometries in $O\left(\mathfrak{b}_{i} M_{i}\right)$ satisfy the congruences defining $O(f)$. There are three cases.

Case 1. Assume that $g<k \leqslant i$. Then $M_{i}$ is an orthogonal summand of $M\left(\mathfrak{F}_{k}\right)$ and

$$
\mathfrak{b}_{i} M_{i} \subseteq \mathfrak{a}_{g i} M_{i} \subseteq \mathfrak{a}_{g k}\left(\mathfrak{g}_{k}: \mathfrak{g}_{i}\right) M_{i} \subseteq a_{g k}\left(\mathfrak{g}_{k}: \mathfrak{g}_{g}\right)\left(\mathfrak{g}_{g} M^{\#}+M\right)
$$


It follows that the congruence defining $O\left(\mathfrak{b}_{i} M_{i}\right)$ implies the congruences for $O(\mathfrak{f})$.

Case 2. Let $g \leqslant i<k$. Then $M_{i} \cap M\left(\mathfrak{g}_{k}\right)=\left(\mathfrak{g}_{k}: \mathfrak{s}_{i}\right) M_{i}$ and

$$
\begin{aligned}
\left(\mathfrak{I}_{k}: \mathfrak{S}_{i}\right) \mathfrak{b}_{i} M_{i} & \cong\left(\mathfrak{g}_{k}: \mathfrak{I}_{i}\right) \mathfrak{a}_{i i+1} M_{i} \\
& \cong a_{g k}\left(\mathfrak{g}_{k}: \mathfrak{S}_{i}\right) M_{i} \\
& \cong \mathfrak{a}_{g k}\left(\mathfrak{g}_{k}: \mathfrak{I}_{g}\right)\left(\mathfrak{g}_{g} M^{\sharp}+M\right),
\end{aligned}
$$

so that the required congruence follows.

Case 3. Let $i<g<k$. Again $M_{i} \cap M\left(\mathfrak{g}_{k}\right)=\left(\mathfrak{g}_{k}: \mathfrak{g}_{i}\right) M_{i}$ and

$$
\left(\mathfrak{g}_{k}: \mathfrak{S}_{i}\right) \mathfrak{b}_{i} M_{i} \subseteq \mathfrak{a}_{g k}\left(\mathfrak{g}_{k}: \mathfrak{g}_{g}\right)\left(\mathfrak{g}_{g} M^{\#}+M\right)
$$

by a modification of the argument in Case 2 , and the necessary congruences follow immediately.

THEOREM 2.7. Let $\mathfrak{f}=\left\{a_{i j}\right\}$ be a semitableau. Then

$$
O(\mathfrak{f})=E(\mathfrak{f}) \prod_{k=1}^{h} O\left(\mathfrak{b}_{k} M_{k}\right)
$$

Proof. We have already established that the right hand group is contained in $O(\mathfrak{f})$. For the converse we generalize the argument of Lemma 2.1. Take $\varphi$ in $O(\mathfrak{f})$. For an element $x$ in an orthogonal basis of $M_{h}$, let

$$
\varphi(x)=\sum_{i=1}^{h} z_{i}
$$

where $z_{i} \in M_{i}, 1 \leqslant i \leqslant h$. Since $B(x, M) \subseteq z_{h}$, it follows that $z_{i}$ is in $\left(\Xi_{h}: \Xi_{i}\right) M_{i}$. In particular, for $i \neq h$,

$$
\text { ord } q\left(z_{i}\right) \geqslant 2\left(e_{h}-e_{i}\right)+e_{i}>e_{h} .
$$

By Hensel's lemma there exists $\beta_{i}$ in $F$ with ord $\beta_{i}=-e_{h}$ such that

$$
\beta_{i}^{2} q\left(z_{h}\right) q\left(z_{i}\right)+2 \beta_{i} q\left(z_{h}\right)=1 .
$$

The component of $E\left(z_{h}, \beta_{i} z_{i}\right) \varphi(x)$ in $M_{i}$ is now zero (for $i \neq h$ ). Here $E\left(z_{h}, \beta_{i} z_{i}\right)$ lies in $E_{i h}\left(\mathrm{a}_{i h}\right)$ since $z_{h}$ is in $M\left(z_{h}\right)$ and, taking $g=i$ and $k=h$ in the congruences defining $O(\mathfrak{f})$, we find that $\beta_{i} z_{i}$ is in $a_{i h} g_{i}^{-1} M_{i}$. Thus, changing $\varphi$ by isometries from $E(\mathfrak{f})$, we can assume $\varphi(x)=z_{h}$ is in $M_{h}$.

Since $\varphi$ is an isometry, $q(x)=q\left(z_{h}\right)$ and, from the congruences defining $O(\mathfrak{f})$, 


$$
x \equiv z_{h} \bmod a_{h-1 h} M_{h} .
$$

As in the proof of Lemma 2.1, there now exists an isometry in $O\left(\mathfrak{b}_{h} M_{h}\right)$ sending $z_{h}$ into $x$. Thus, using isometries in $E(\mathfrak{f})$ and $O\left(\mathfrak{b}_{h} M_{h}\right)$, we can change $\varphi$ into an isometry acting identically on $M_{h}$. Proceeding in this manner, the proof is easily completed.

REMARK 2.8. It follows from the proof of Theorem 2.7 that $O(f)$ is generated by isometries of the form $E(x, y)$ where $x \in M_{j}$ and $y \in a_{i j} \xi_{i}^{-1} M_{i}$ for $1 \leqslant i$ $<j \leqslant h$, together with isometries in $O\left(\mathfrak{b}_{k} M_{k}\right), 1 \leqslant k \leqslant h$.

THEOREM 2.9. Let $f=\left\{a_{i j}\right\}$ be a semitableau. Then $E(\mathfrak{f})$ is the set of those isometries $\varphi$ in $O(f)$ which satisfy the congruences

$$
\varphi(x) \equiv x \bmod \left(\mathfrak{a}_{k-1 k}\left(\mathfrak{g}_{k}: \mathfrak{g}_{k-1}\right) M+\mathfrak{a}_{k k+1} \mathfrak{g}_{k+1} M^{\sharp}\right)
$$

for all $x$ in $M\left(\mathfrak{g}_{k}\right), 1 \leqslant k \leqslant h$.

Proof. We show first that any $\varphi$ in $E(\mathfrak{f})$ satisfies the above congruences. Recall from Lemma 2.5 that any $\varphi_{i j}$ in $E_{i j}\left(a_{i j}\right)$ satisfies

$$
\varphi_{i j}(x) \equiv x \bmod N
$$

for all $x$ in $M\left(\mathfrak{g}_{k}\right)$, where

$$
N=\mathfrak{a}_{i j} \mathfrak{g}_{i}^{-1}\left(\left(\mathfrak{g}_{k} \cap \mathfrak{g}_{j}\right) M\left(\mathfrak{g}_{i}\right)+\left(\mathfrak{g}_{k} \cap \mathfrak{g}_{i}\right) M\left(\mathfrak{g}_{j}\right)\right) .
$$

Case 1. Let $i<j \leqslant k$. Then

$$
N=\mathfrak{a}_{i j}\left(\mathfrak{g}_{k}: \mathfrak{s}_{i}\right) M\left(\mathfrak{g}_{i}\right) \subseteq \mathfrak{a}_{i k}\left(\mathfrak{g}_{k}: \mathfrak{g}_{i}\right) M \leqq \mathfrak{a}_{k-1 k}\left(\mathfrak{g}_{k}: \mathfrak{g}_{k-1}\right) M .
$$

Case 2. Let $i<k<j$. Then

$$
\begin{aligned}
N & =\mathfrak{a}_{i j}\left(\mathfrak{g}_{j}: \mathfrak{g}_{i}\right) M\left(\mathfrak{g}_{i}\right)+\mathfrak{a}_{i j}\left(\mathfrak{g}_{k}: \mathfrak{g}_{i}\right) M\left(\mathfrak{g}_{j}\right) \\
& \subseteq \mathfrak{a}_{i j}\left(\mathfrak{g}_{j}: \mathfrak{g}_{i}\right) M+\mathfrak{a}_{k k+1} \mathfrak{g}_{k+1}\left(\mathfrak{g}_{j}^{-1} M\left(\mathfrak{g}_{j}\right)\right) \\
& \subseteq \mathfrak{a}_{k-1 k}\left(\mathfrak{g}_{k}: \mathfrak{g}_{k-1}\right) M+\mathfrak{a}_{k k+1} \mathfrak{g}_{k+1} M^{\sharp} .
\end{aligned}
$$

Case 3. Let $k \leqslant i<j$. Then

$$
N=a_{i j} M\left(\mathfrak{g}_{j}\right) \leqq a_{k j} \mathfrak{g}_{j} M^{\#} \leqq a_{k k+1} \mathfrak{g}_{k+1} M^{\sharp} .
$$

The congruences in the statement of the theorem now follow immediately. To prove, conversely, that any isometry in $O(f)$ satisfying these congruences is in $E(\mathrm{f})$, it suffices by the previous theorem to study isometries in $O\left(M_{k}\right), 1 \leqslant k$ $\leqslant h$. But for $\varphi$ in $O\left(M_{k}\right)$ satisfying these congruences, we have 


$$
\varphi(x) \equiv x \bmod c_{k} M_{k}
$$

for all $x$ in $M_{k}$. Since by Lemma 2.3,

$$
O\left(\mathfrak{a}_{k-1 k}\left(\mathfrak{g}_{k}: \mathfrak{a}_{k-1}\right) M_{k}\right) \subseteq E_{k-1 k}\left(\mathfrak{a}_{k-1 k}\right) \subseteq E(\mathfrak{f})
$$

and

$$
O\left(\mathfrak{a}_{k k+1}\left(\mathfrak{g}_{k+1}: \mathfrak{s}_{k}\right) M_{k}\right) \subseteq E_{k k+1}\left(\mathfrak{a}_{k k+1}\right) \subseteq E(\mathfrak{f}),
$$

it follows that all such isometries are in $E(\mathfrak{f})$.

THEOREM 2.10. Let $\mathfrak{f}=\left\{a_{i j}\right\}$ be a semitableau. Then

$$
\frac{O(\mathfrak{f})}{E(\mathfrak{f})} \cong \prod_{k=1}^{h} \frac{O\left(\mathfrak{b}_{k} M_{k}\right)}{O\left(\mathfrak{c}_{k} M_{k}\right)}
$$

Proof. The theorem follows from the two previous theorems and their proofs.

TheOREM 2.11. Let $\mathfrak{f}=\left\{a_{i j}\right\}$ be a semitableau. Then $E(\mathfrak{f})$ is generated by isometries of the form

$$
E(u, v) E(x, y) E(u, v)^{-1}
$$

where $u, x \in M_{j}, y \in a_{i j} \mathfrak{g}_{i}^{-1} M_{i}$ and $v \in \mathfrak{g}_{i}^{-1} M_{i}, 1 \leqslant i<j \leqslant h$.

Proof. By Remark 2.8 and Theorem 2.10, it suffices to show that the subgroups $O\left(c_{k} M_{k}\right)$ are generated by the given isometries for those $k$ for which the rank of $M_{k}$ is at least two. From the definition of $c_{k}$, it is enough to verify that both $O\left(\mathfrak{a}_{k-1 k}\left(\mathfrak{a}_{k}: \mathfrak{a}_{k-1}\right) M_{k}\right)$ and $O\left(\mathfrak{a}_{k k+1}\left(\mathfrak{g}_{k+1}: \mathfrak{g}_{k}\right) M_{k}\right)$ are generated by the given isometries. We prove the result for the first group; a slight modification of the argument will handle the second group.

Take $z$ in $\mathfrak{g}_{k-1}^{-1} M_{k-1}, r$ in $M_{k}, s$ in $a_{k-1 k} M_{k}$ with $B(r, s)=0$, and let $t$ in $M_{k}$ be determined by the equation

$$
(1+q(z) q(r)) t+2 q(z) s \otimes r \otimes t=(1-q(z) q(r)) s .
$$

Then $t$ is in $a_{k-1 k} M_{k}$,

$$
q(t)(1+q(z) q(r)+2 q(z) s \otimes r)=(1-q(z) q(r)) s \otimes t
$$

and

$$
\begin{aligned}
(1+z \otimes r) \otimes & (1+z \otimes s) \otimes(1-z \otimes r) \otimes(1-z \otimes t) \\
& =(1+q(z) q(t))(1+q(z) q(r)+2 q(z) s \otimes r) .
\end{aligned}
$$

It follows that 


$$
E(z, r) E(z, s) E(z, r)^{-1} E(z,-t)=E(\beta s, r)
$$

where $\beta=2 q(z)(1+q(z) q(r))^{-1}$ is an element in $\xi_{k-1}^{-1}$. The result now follows immediately from Lemma 2.1. For the other case, take $z$ in $\mathfrak{s}_{k}^{-1} M_{k+1}$; the rest of the proof is similar.

REMARK 2.12. To conclude this section we compare Riehm's tableau with our semitableau. A tableau $\left[10\right.$, p. 554] $\mathfrak{t}=\left\{\mathfrak{g}_{i j}\right\}$ is a family of ideals $g_{i j}, 1 \leqslant i, j \leqslant h$, satisfying various conditions. In particular, $\mathfrak{g}_{i} g_{j i}=g_{j} g_{i j}$, so that only the $g_{i j}$ with $i \leqslant j$ make an essential contribution. Other conditions force the subset $\left\{g_{i j} \mid i<j\right\}$ to coincide with our semitableau. Thus only the ideals $\mathrm{g}_{i i}, 1 \leqslant i \leqslant h$, make a new contribution. The remaining conditions on the tableau force $\mathrm{c}_{i} \subseteq \mathrm{g}_{i i} \subseteq \mathfrak{b}_{i}$ for $1 \leqslant i \leqslant h$, where the ideals $\mathfrak{b}_{i}$ and $\mathrm{c}_{i}$ are as we defined for a semitableau. This indicates why Riehm's classification using tableaux cannot be extended if there are Jordan components of rank 1 or 2 . If rank $M_{i}=1$, there are too many ideals $g_{i i}$, since the group $O\left(M_{i}\right)$ has only two elements; while, if rank $M_{i}=2$, the ideals $g_{i i}$ are not enough to describe all the normal subgroups in $O\left(\mathfrak{b}_{i} M_{i}\right) / O\left(\mathrm{c}_{i} M_{i}\right)$. However, for rank $M_{i} \geqslant 3$, the normal subgroups in $O\left(M_{i}\right)$ are essentially congruence subgroups and therefore correspond to the $g_{i i}$.

3. Open normal subgroups. We now study the open normal subgroups of $O(M)$ when the reside class field of $F$ is finite, of characteristic $p$ say, and has at least five elements. Assume also, since otherwise the result is well known [5], that $\operatorname{dim} V \geqslant 3$ and that $M$ has at least two Jordan components. It can be shown that any noncentral normal subgroup in $O(M)$ is open in the $\mathfrak{p}$-adic topology. For example, if $V$ is isotropic, the argument in [2] can be modified (and greatly simplified) to show that every noncentral normal subgroup in $O(M)$ contains a nontrivial congruence subgroup and consequently is open. Again, the methods of this paper can be used to establish the result, but we shall not include a proof. See also Riehm [11] for a very general result in this direction.

Let $\Psi_{i}, 1 \leqslant i \leqslant h-1$, be the isometry defined by the conditions $\Psi_{i}(x)$ $=x$ for $x \in M_{k}$ with $k \leqslant i$, and $\Psi_{i}(x)=-x$ for $x \in M_{k}$ with $k>i$. For a semitableau $\mathfrak{f}=\left\{\mathfrak{a}_{i j}\right\}$, let $I(\mathfrak{f})$ be the subgroup of isometries generated by $-I$ and those $\Psi_{i}$ for which $a_{i i+1}=0$. Then $I(\mathfrak{f})$ is a finite abelian group with exponent two. Moreover, for almost all semitableaux, $I(\mathfrak{f})=\{ \pm I\}$. In general, card $I(\mathfrak{f}) \leqslant 2^{h}$.

This section will be devoted to proving the following result.

MAIN THEOREM. Let $F$ be a local field with a residue class field of at least 5 elements. If $F$ is not a p-adic number field ( $p$ a rational prime), assume that there is at most one Jordan component of $M$ with rank 1 . Then any open normal subgroup $\mathfrak{R}$ of $O(M)$ satisfies 


\section{$E(\mathfrak{f}) \subseteq \mathfrak{N} \subseteq I(\mathfrak{f}) O(\mathfrak{f})$}

for a suitable unique semitableau $\mathfrak{f}$.

The proof will be broken into parts. We start by constructing $\mathfrak{f}$. For each $i$, $j$ with $1 \leqslant i<j \leqslant h$, let $\mathfrak{a}_{i j}$ be an ideal maximal such that $E_{i j}\left(\mathfrak{a}_{i j}\right)$ is in $\mathfrak{R}$. Since $\mathfrak{N}$ is open and hence contains a congruence subgroup, $a_{i j} \neq 0$. It follows from Lemma 2.2 and the maximality of the $a_{i j}$ that $\mathfrak{f}=\left\{a_{i j}\right\}$ is a semitableau. Clearly,

$$
E(\mathfrak{f})=\prod_{i<j} E_{i j}\left(\mathfrak{a}_{i j}\right) \subseteq \mathfrak{R}
$$

The difficult part is to establish that $\mathfrak{R} \subseteq I(\mathfrak{f}) O(\mathfrak{f})$. However, it is easily seen that $\mathfrak{f}$ is unique for a given $\mathfrak{R}$. For suppose there also exists $\mathfrak{f}^{\prime}=\left\{a_{i j}^{\prime}\right\}$ with

$$
E\left(\mathfrak{f}^{\prime}\right) \subseteq \Re \subseteq I\left(\mathfrak{f}^{\prime}\right) O\left(\mathfrak{f}^{\prime}\right)
$$

and that $a_{g k}^{\prime} \subsetneq a_{g k}$ for some $g<k$. Take $r$ in $a_{g k} g_{g}^{-1} M_{g}$ and $s, x$ in $M_{k}$ with ord $B(x, s)=e_{k}$. Then

$$
E(r, s) \in E_{g k}\left(\mathfrak{a}_{g k}\right) \subseteq E(\mathfrak{f}) \subseteq \mathfrak{N} \subseteq I\left(\mathfrak{f}^{\prime}\right) O\left(\mathfrak{f}^{\prime}\right)
$$

but since

$$
E(r, s)(x)=x+(1+q(r) q(s))^{-1} B(x, s)(r-q(r) s),
$$

$\pm E(r, s)$ does not satisfy the $(g, k)$-congruence in the definition of $O\left(\mathfrak{f}^{\prime}\right)$.

Now fix an open normal subgroup $\mathfrak{N}$ of $O(M)$ and let $f=\left\{a_{i j}\right\}$ be the semitableau constructed above. Thus $E(\mathfrak{f}) \subseteq \mathfrak{N}$. The rest of this section is devoted to proving $\mathfrak{N} \subseteq I(\mathfrak{f}) O(\mathfrak{f})$.

LEMMA 3.1. Let $E(x, t)^{q} \in \mathfrak{N}$ where $(p, q)=1$ and $q(x) q(t) \in \mathfrak{p}$. Then $E(x, t)$ is in $\mathfrak{R}$.

Proof. For $P=p^{d}$ and $d$ large, choose positive integers $c$ and $m$ such that $q m-c P=1$. Since $E(x, t)^{q m}$ is in $\Re$, it is enough to prove that $E(x, t)^{P}$ is in $\mathfrak{N}$. From the identity

$$
(1+x \otimes t)^{n}=1+n x \otimes t-\left(\begin{array}{l}
n \\
2
\end{array}\right) q(x) q(t)-\left(\begin{array}{l}
n \\
3
\end{array}\right) q(x) q(t) x \otimes t+\cdots
$$

for a positive integer $n$, it is easily seen that $E(x, t)^{n}=E(x, \nu t)$ with $\nu$ $\equiv n \bmod p$. It follows, for $d$ large, that $E(x, t)^{P}$ can be taken arbitrarily close to the identity map, and hence lies in the open subgroup $\Re$.

LEMMA 3.2. Let $M_{h}=\mathrm{ox} \perp N$ for some $x$ in $M_{h}$. Then any isometry $\varphi$ in $O(M)$ can be expressed as 


$$
\varphi=\theta E\left(x, t_{1}\right) \cdots E\left(x, t_{h-1}\right) \psi
$$

where $\theta$ is in $O\left(M_{h}\right), t_{i} \in \mathfrak{\Im}_{i}^{-1} M_{i}, 1 \leqslant i \leqslant h-1$, and $\psi$ is in $O(M)$ with $\psi(x)$ $=x$. Moreover, if $\varphi$ is in $\mathfrak{R}$, then $\theta \psi$ and all the $E\left(x, t_{i}\right)$ are in $\mathfrak{N}$.

Proof. The first part follows as in the proof of Theorem 2.7. Let $\varphi(x)$ $=\sum_{i=1}^{h} z_{i}$ where $z_{i} \in M_{i}, 1 \leqslant i \leqslant h$. As in Theorem 2.7 , choose $\beta_{1}$ such that in $E\left(z_{h}, \beta_{1} z_{1}\right) \varphi(x)$ the $M_{1}$ component is zero. Proceeding in this manner, choose the $\beta_{i}$ such that

$$
E\left(z_{h}, \beta_{h-1} z_{h-1}\right) \cdots E\left(z_{h}, \beta_{1} z_{1}\right) \varphi(x)=\varepsilon z_{h}
$$

where $\varepsilon$ is a unit in 0 . Here $\beta_{i} z_{i} \in \mathfrak{g}_{i}^{-1} M_{i}, 1 \leqslant i \leqslant h-1$. Since $q(x)$ $=q\left(\varepsilon z_{h}\right)$, there exists $\theta$ in $O\left(M_{h}\right)$ such that $\theta(x)=\varepsilon z_{h}$. Let $t_{i}=-\varepsilon^{-1} \beta_{i} z_{i}$ and

$$
\begin{aligned}
\psi & =\theta^{-1} E\left(z_{h}, \beta_{h-1} z_{h-1}\right) \cdots E\left(z_{h}, \beta_{1} z_{1}\right) \varphi \\
& =E\left(x,-t_{h-1}\right) \cdots E\left(x,-t_{1}\right) \theta^{-1} \varphi .
\end{aligned}
$$

Then $\psi(x)=x$ and the given decomposition of $\varphi$ follows.

Now assume $\varphi$ is in $\mathfrak{R}$. Let $\Psi(x)$ denote the symmetry about $x$. Then the commutator

$$
[\varphi, \Psi(x)]=\theta E\left(x, t_{1}\right) \cdots E\left(x, t_{h-1}\right) E\left(x, t_{h-1}\right) \cdots E\left(x, t_{1}\right) \Psi(x) \theta^{-1} \Psi(x)
$$

is also in $\mathfrak{N}$. If $-I_{h-1}$ denotes the negative identity mapping in $O\left(M_{h-1}\right)$, then

$$
\left[[\varphi, \Psi(x)],-I_{h-1}\right]=\tau E\left(x, t_{h-1}\right)^{4} \tau^{-1}
$$

is in $\mathfrak{N}$ where $\tau=\theta E\left(x, t_{1}\right) \cdots E\left(x, t_{h-2}\right)$. It follows that $E\left(x, t_{h-1}\right)^{4}$, and hence also $E\left(x, t_{h-1}\right)$, is in $\Re$. The rest of the proof is clear.

LEMMA 3.3. Let $E(x, u)$ and $E(x, v)$ be in $\mathfrak{N}$ with $q(x) q(u)$, $q(x) q(v)$ and $q(x) B(u, v)$ in p. Define $w$ by

$$
w-u-v=q(x) w \otimes u \otimes v .
$$

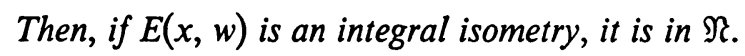

Proof. Because of the conditions on $x, u$ and $v$, the equation

$$
w \otimes(1-q(x) u \otimes v)=u+v
$$

can be solved for $w$ after multiplying on the right by $1-q(x) v \otimes u$. Moreover, $B(x, w)=0$. Multiplying the equation for $w$ on the right by $v$ gives

$$
w \otimes v-u \otimes v=q(v)(1+q(x) w \otimes u) .
$$

Hence 
$(1-x \otimes w) \otimes(1+x \otimes u) \otimes(1+x \otimes v)=(1+q(x) q(v))(1+q(x) w \otimes u)$

and consequently

$$
E(x, u) E(x, v)=E(x, w) \theta
$$

is in $\mathfrak{N}$, where $\theta$ is the isometry induced by an inner automorphism with $1+q(x) w \otimes u$. In particular, $\theta(x)=x$. After conjugating by $\Psi(x)$, it follows that $E(x, w)^{2}$, and hence also $E(x, w)$, is in $\Re$.

Remark 3.4. The next sequence of arguments will show for each $E\left(x, t_{i}\right)$ constructed from $\varphi \in \mathfrak{N}$ in Lemma 3.2, there exists a semitableau, $\mathfrak{f}_{i}$ say, such that $E\left(x, t_{i}\right) \in E\left(\mathfrak{f}_{i}\right) \cong \mathfrak{R}$. Then, to complete the proof of the main theorem, by repeated application of Lemma 3.2, it is enough to show only those $\varphi$ in $\mathfrak{R}$ of the form $\varphi=\theta_{1} \cdots \theta_{h}$ with $\theta_{i}$ in $O\left(M_{i}\right), 1 \leqslant i \leqslant h$, are in $I(\mathfrak{f}) O(\mathfrak{f})$. This final step will then easily follow.

Definition. Let $L$ be a modular orthogonal summand of $M$ with scale $\Xi L=\Im$ and let $x \in V$ with $B(x, L)=0$ be such that $E(x, y)$ is an isometry in $O(M)$ for all $y$ in $L$. For an ideal $\mathfrak{b} \neq 0$ in $\mathfrak{0}$, define $S(x, \mathfrak{b} L)$ to be the set of all $r$ in $\mathfrak{b} L$ such that $E(x, r)$ is in $\Re$.

Starting from $E(x, t)$ in $\mathfrak{N}$, by studying the size of $S(x, \mathfrak{b} L)$ and using Theorem 2.11, we will be able to prove Remark 3.4. The sets $S(x, \mathfrak{b} L)$ have the following properties.

(a) There exists an ideal $\mathfrak{a} \neq 0$ such that $\mathfrak{a} L \cong S(x, \mathfrak{b} L)$.

PROoF. Since $\mathfrak{R}$ is open, it contains a nontrivial congruence subgroup and hence all $E(x, r) \in \mathfrak{R}$ for $r \in \mathfrak{a} L$, provided $\mathfrak{a}$ is sufficiently small.

(b) $\theta(S(x, \mathfrak{b} L))=S(x, \mathfrak{b} L)$ for all $\theta$ in $O(L)$.

Proof. Conjugate $E(x, r)$ in $\mathfrak{R}$ by $\theta$ in $O(L)$.

(c) If $u \in S(x, \mathfrak{b} L)$ and $v \in S(x, \mathfrak{b c} L)$ for ideals $\mathfrak{b}$ and $\mathfrak{c}$ in $\mathfrak{0}$, then there exists $w$ in $S(x, \mathfrak{b} L)$ such that $w \equiv u+v \bmod q(x) \mathfrak{b}^{3} \mathfrak{c} L$, provided $q(x) \mathfrak{s b}^{2} \mathfrak{c} \leqq \mathfrak{p}$.

Proof. The result follows immediately for the $w \in \mathfrak{b} L$ in Lemma 3.3.

(d) Let $F$ be the field of $p$-adic numbers $(p \neq 3$ a rational prime). If $t$ is in $S(x, \mathfrak{b} L)$ and $q(x) q(t) \in \mathfrak{p}$, then $\mathrm{o} t \leqq S(x, \mathfrak{b} L)$.

PROOF. It is easily verified, with the help of the identity in the proof of Lemma 3.1, that the cyclic subgroup generated by $E(x, t)$ in the closed group $\mathfrak{N}$ is dense in the subgroup $\{E(x, \alpha t) \mid \alpha \in \mathfrak{0}\}$ of $O(M)$, provided $q(x) q(t) \in \mathfrak{p}$.

(e) Let rank $L \geqslant 2$ and $\mathfrak{p b} L \subsetneq S(x, \mathfrak{b} L)$. Then, if $q(x) \mathfrak{s} \mathfrak{b}^{2} \subseteq \mathfrak{p}$,

$$
S(x, \mathfrak{b} L)=\mathfrak{b} L
$$

Proof. By hypothesis, there exists $E(x, s)$ in $\mathfrak{R}$ with $s$ in $\mathfrak{b} L$, but not in $\mathfrak{p b} L$. Let $o r$ be an orthogonal summand of $L$. By (b), $\Psi(r)(s)$ is in $S(x, \mathfrak{b} L)$, where $\Psi(r)$ is the symmetry about $r$. Using (c), there now exists $w$ in $S(x, \mathfrak{b} L)$ with 


$$
w \equiv s-\Psi(r)(s) \equiv q(r)^{-1} B(r, s) r \bmod \mathfrak{p b} L .
$$

Since $\mathfrak{k b} L \subseteq S(x, \mathfrak{b} L)$, it follows by repeated use of (c) that $q(r)^{-1}$. $B(r, s) r$ is in $S(x, \mathfrak{b} L)$.

If $\mathfrak{b}^{-1} s$ is not an orthogonal summand of $L$, choose the $r$ above such that $q(r)^{-1} B(r, s)$ generates $\mathfrak{b}$, and replace $s$ by $q(r)^{-1} B(r, s) r$. We therefore assume $\mathfrak{b}^{-1} s$ splits $L$.

Fix a generator $\beta$ of $\mathfrak{b}$. Let $t$ be a primitive element of $L$ orthogonal to $s$ such that $r=\beta^{-1} s+t$ generates an orthogonal summand of $L$. As above, $q(r)^{-1} B(r, s) r$ is in $S(x, \mathfrak{b} L)$. Replacing $t$ by $-t$ and subtracting gives $2 q(r)^{-1} B(r, s) t$ is in $S(x, \mathfrak{b} L)$. The coefficient $2 q(r)^{-1} B(r, s)$, or $4 \beta q\left(\beta^{-1} s\right) q(r)^{-1}$, generates the ideal $\mathfrak{b}$. Now count the possible coefficients of $t$ in $\mathfrak{b}$ modulo $\mathfrak{p b}$ that can be obtained by this method. Replacing $t$ by scalar multiples of $t$, we find there are enough distinct coefficients modulo $\mathfrak{p b}$ to generate $\mathfrak{b} / \mathfrak{p b}$ as an additive abelian group. Since $\mathfrak{p b} L \subseteq S(x, \mathfrak{b} L)$, it now follows from (c) that $\mathfrak{b} t \leqq S(x, \mathfrak{b} L)$. This is easily extended to $\mathfrak{b} L \subseteq S(x, \mathfrak{b} L)$. The converse inclusion is obvious.

REMARK 3.5. (e) above is very important for the proof of the main theorem. The counting argument uses $5 \leqslant$ card $o / \mathfrak{p}<\infty$. For most residue class fields, for example if $o / \mathfrak{p}$ is any local or global field, (e) is false, and the main theorem does not hold as stated. However, if $o / p$ is the field of real numbers, the coefficients of $t$ in the above proof will include an euclidean neighborhood of zero (in $\mathfrak{b} / \mathfrak{p b} \cong \mathbf{R}$ ) and this is enough to generate $\mathfrak{b} / \mathfrak{p b}$ as an additive group. This would enable us to also handle the case studied by Pollak [9] and Martin [6], since it is not difficult to circumvent the other places we have used properties of $\mathfrak{o} / \mathfrak{p}$.

(f) Let rank $L \geqslant 2$ and $s \in S(x, \mathfrak{b} L)$ but $s \notin \mathfrak{p b} L$. Then $\mathfrak{b} L=S(x, \mathfrak{b} L)$, provided $q(x) \mathfrak{s b}^{2} \subseteq \mathfrak{p}$.

Proof. First, as in (e), we may assume $\mathfrak{b}^{-1} s$ is an orthogonal summand of $L$. From (a), (e) and induction, it suffices to prove there exists $E(x, y)$ in $\mathfrak{N}$ where $(\mathrm{pb})^{-1} y$ is an orthogonal summand of $L$. Choose $t$ in $L$ such that ot is an orthogonal summand of $L$ and $q(t)^{-1} B(s, t)$ generates the ideal $\mathrm{pb}$. As in Lemma 3.3, define $y$ by the condition $E(x, y) E(x, s) E(x,-\Psi(t)(s))$ is in $O(L)$. Then, as in (c),

$$
y \equiv-q(t)^{-1} B(s, t) t \bmod \mathfrak{p}^{2} \mathfrak{b} L
$$

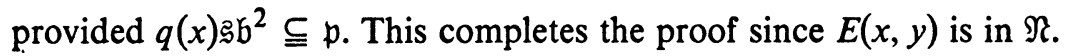

We establish now the result mentioned in Remark 3.4 using these properties of $S(x, \mathfrak{b} L)$ by taking $L$ to be the appropriate $M_{i}$ or $\xi_{i}^{-1} M_{i}$. By assumption, all but at most one $M_{i}$ has rank at least two, except when $F$ is a $p$-adic number field. In the exceptional case, (d) can be used instead of (f). 
Lemma 3.6. Assume the hypothesis of the main theorem. Let $E(x, t)$ be in $\mathfrak{R}$ where $\mathrm{ox}$ is an orthogonal summand of $M_{k}$ and $t \in \mathfrak{s}_{g}^{-1} M_{g}$ for some $g<k$. Then there exists a semitableau $\mathfrak{f}^{*}$ such that $E(x, t) \in E\left(f^{*}\right) \cong \mathfrak{N}$.

Proof. Let $a$ be the ideal for which $t$ is primitive in $\mathfrak{a}_{g}^{-1} M_{g}$ and $f^{*}=\left\{a_{i j}^{*}\right\}$ the semitableau in Example 2.4 for the ideal $a$ and indices $g, k$. By Theorem 2.11 and (f) (or (d)), to complete the proof it suffices to show for each $i<j$ that $\mathfrak{R}$ contains an isometry of the form $E\left(x_{j}, t_{i}\right)$ where $t_{i}$ and $x_{j}$ are primitive in $\mathrm{a}_{i j}^{*} g_{i}^{-1} M_{i}$ and $M_{j}$, respectively. We already have the case $i=g, j=k$ and may, therefore, also assume $a^{-1} t$ is an orthogonal summand of $g_{g}^{-1} M_{g}$.

We establish now the case $i<g$ and $j=k$. The method is then easily adapted to the other cases. Let $v$ be primitive in $\mathfrak{a}_{i}^{-1} M_{i}$. Then the symmetry $\Psi(t+v)$ is an integral isometry which maps $t$ into an element of the form $u=\varepsilon t+\varepsilon^{\prime} v$ in $a_{g}^{-1} M\left(\mathfrak{g}_{g}\right)$ with $\varepsilon$ and $\varepsilon^{\prime}$ units. Consequently, both $E(x, \varepsilon t)$ and $E(x, u)$ are in $\mathfrak{R}$. Now, by Lemma $3.3, E(x, s)$ is in $\mathfrak{R}$ where $s-\varepsilon t+u$ $=-\varepsilon q(x) s \otimes t \otimes u$. Simplifying, we find $s=\zeta v+\lambda t$ where $\zeta$ is a unit and $\lambda \in \mathfrak{z}_{k} \xi_{i}{ }^{-1}$.It is not difficult to find an integral isometry in $O\left(M_{i} \perp M_{g}\right)$ which sends $s$ into $\eta v, \eta$ a unit. Thus $E(x, \eta v)$ is in $\Re$, constructing the required isometry with $t_{i}=\eta v$. This completes the proof.

LEMMA 3.7. Let $x_{1}, \ldots, x_{n}$ be an orthogonal basis of the $s_{k}$-modular lattice $M_{k}$. Then any isometry $\theta$ in $O\left(M_{k}\right)$ can be expressed in the form

$$
\boldsymbol{\theta}=E\left(x_{1}, t_{1}\right) \cdots E\left(x_{n-1}, t_{n-1}\right) \chi
$$

where $t_{i}$ is in $g_{k}^{-1} M_{k}$ with $B\left(x_{i}, t_{j}\right)=0$ for $1 \leqslant i \leqslant j \leqslant n-1$, and $\chi$ is an isometry which fixes the basis elements except for sign.

PRoof. Assume rank $M_{k} \geqslant 2$, for otherwise the result is trivial. Let $\theta\left(x_{1}\right)$ $=\alpha x_{1}+z$ where $B\left(x_{1}, z\right)=0$. Since $q\left(\alpha x_{1}+z\right)=q\left(x_{1}\right)$, the equation

$$
\beta^{2} q\left(x_{1}\right) q(z)+2 \alpha \beta q\left(x_{1}\right)=1
$$

has roots $\beta=q(z)^{-1}(-\alpha \pm 1)=q\left(x_{1}\right)^{-1}(\alpha \pm 1)^{-1}$. For at least one choice of sign, $\alpha \pm 1$ is a unit. Then

$$
1+q\left(x_{1}\right) q(\beta z)=2(1 \pm \alpha)^{-1}
$$

is also a unit, so that $E\left(x_{1}, \beta z\right)$ is a well-defined isometry in $O\left(M_{k}\right)$. Moreover, $E\left(x_{1}, \beta z\right) \theta\left(x_{1}\right)= \pm x_{1}$. Repeating this argument on the orthogonal complement of $o x_{1}$, we can reduce $\theta$ to an isometry $\chi$ in $O\left(M_{k}\right)$ where $\chi\left(x_{i}\right)= \pm x_{i}$, $1 \leqslant i \leqslant n$.

Proof OF MAIN THeOReM. We have already constructed $E(\mathfrak{f}) \cong \mathfrak{N}$. Now, for $\varphi$ in $\mathfrak{R}$, we must prove $\varphi$ is in $I(\mathfrak{f}) O(\mathfrak{f})$. By Lemmas 3.2 and 3.6 and Remark 3.4 , it suffices to consider only $\varphi$ of the form 


$$
\varphi=\theta_{1} \theta_{2} \cdots \theta_{h}
$$

with $\theta_{k}$ in $O\left(M_{k}\right), 1 \leqslant k \leqslant h$.

If $\mathfrak{f}=\left\{\mathfrak{a}_{i j}\right\}$ and $\mathfrak{b}_{k}=a_{k-1 k} \cap \mathfrak{a}_{k k+1}$, we show first that $\pm \theta_{k}$ is in $O\left(\mathfrak{b}_{k} M_{k}\right)$, $1 \leqslant k \leqslant h$, for at least one choice of sign. This is obviously true if $\mathfrak{b}_{k}=\mathfrak{o}$ or rank $M_{k}=1$. Fix $k$ and, assuming $\mathfrak{b}_{k} \subseteq \mathfrak{p}$ and rank $M_{k} \geqslant 2$, let

$$
\theta_{k}=E\left(x_{1}, t_{1}\right) \cdots E\left(x_{n-1}, t_{n-1}\right) \chi_{k}
$$

with $x_{i}, t_{i}$ and $\chi_{k}$ as in Lemma 3.7. Then the commutator

$$
\tau=\left[\varphi, \Psi\left(x_{1}\right)\right]=\left[\theta_{k}, \Psi\left(x_{1}\right)\right]=E\left(x_{1}, t_{1}\right)^{2}
$$

is in $\mathfrak{R}$. Let the ideal $\delta$ be minimal such that $E\left(x_{1}, t_{1}\right)$ is in $O\left(\supset M_{k}\right)$. Then $\tau$ is also in $O\left(\delta M_{k}\right)$, but not in $O\left(p d M_{k}\right)$. Choose $r$ in $M_{k}$ such that $\tau(r)-r$ is in $\delta M_{k}$, but not in $\mathfrak{b} M_{k}$. We next show that $\delta \subseteq a_{k-1 k}$ (if $k \geqslant 2$ ). Take $z$ in $\mathfrak{g}_{k-1}^{-1} M_{k-1}$ with ord $q(z)=-e_{k-1}$. Then

$$
[E(z, r), \tau]=E(z, r) E(z,-\tau(r))
$$

is in $\Re$. As in Lemma 3.3, we obtain from this an isometry $E(z, y)$ in $\Re$ where $y \otimes(1+q(z) r \otimes \tau(r))=r-\tau(r)$. Hence $y \in \delta M_{k}$, but $y \notin \mathfrak{p d} M_{k}$. As in Lemma 3.6, $E(z, y) \in E_{k-1 k}(\delta) \subseteq \mathfrak{N}$ and hence $\delta \subseteq a_{k-1 k}$. Similarly, $\delta$ $\subseteq \mathfrak{a}_{k k+1}$, so that $\mathfrak{d} \subseteq \mathfrak{b}_{k}$. Thus $E\left(x_{1}, t_{1}\right)$ is in $O\left(\mathfrak{b}_{k} M_{k}\right)$. Since $E\left(x_{1}, t_{1}\right)^{2}$ is in $\mathfrak{N}$, by Lemma 3.1, $E\left(x_{1}, t_{1}\right)$ is also in $\mathfrak{N}$. Proceeding in this manner shows that $\theta_{k} \chi_{k}$ is in $O\left(\mathfrak{b}_{k} M_{k}\right)$. Since $\mathfrak{b}_{k} \subseteq \mathfrak{p}$ and $\theta_{k}$ is independent of the choice of the basis, we must have $\chi_{k}= \pm I_{k}$, where $I_{k}$ denotes the identity mapping on $M_{k}$.

We now have $\chi_{1} \cdots \chi_{h} \varphi \in O(\mathfrak{f})$ and must show that $\chi_{1} \cdots \chi_{h} \in I(\mathfrak{f})$. Suppose $\chi_{i}=I_{i}$ for $1 \leqslant i \leqslant k$, but $\chi_{k+1}=-I_{k+1}$. It follows in this case that $\mathfrak{a}_{k k+1}=\mathfrak{o}$. For if $\mathfrak{b}_{k}+\mathfrak{b}_{k+1} \subseteq \mathfrak{a}_{k k+1} \subseteq \mathfrak{p}$, let $r$ and $s$ be primitive in $\mathfrak{g}_{k}^{-1} M_{k}$ and $M_{k+1}$, respectively. Then

$$
\sigma=[E(r, s), \varphi]=E(r, s) E\left(\theta_{k}(r),-\theta_{k+1}(s)\right)
$$

is in $\mathfrak{R}$ and, since $\theta_{k}(r) \equiv r \bmod \mathfrak{b}_{k} M_{k}$ and $-\theta_{k+1}(s) \equiv s \bmod \mathfrak{b}_{k+1} M_{k+1}, \sigma$ is approximately $E(r, s)^{2}$. Thus $a_{k k+1}$ must be $\mathfrak{o}$ so that $\Psi_{k}$ is in $I(\mathfrak{f})$. It is now clear how to complete the proof.

REMARK 3.8. We comment now on Theorem 6.5 of Bröcker [1]. In [1] the residue class field is arbitrary but $V$ is an anisotropic space. Thus $O(V)$ $=O(M)$, where $M$ is a maximal lattice on $V$. Hence $M$ is either modular, or has two Jordan components with $\mathfrak{g}_{2}=\mathfrak{p} \mathfrak{g}_{1}$. Now our semitableau $f=\{a\}$ becomes a single ideal. Let $a=\mathfrak{p}^{k}$ and $O_{k}$ be as defined in [1]. Comparing the definitions, we find $O(a)=O_{2 k}$ and $E(a)=O_{2 k+1}$. Theorem 6.5 in [1] states: after projecting out $\{ \pm I\}$, every normal subgroup in $O(V)$ satisfies $P O_{k+1} \subseteq \mathfrak{N}$ 
$\cong P O_{k}$ for some integer $k$. Our result, which uses properties of the finite residue class field (or the real field if we were treating $\mathbf{R}[[X]]$ as in [6], [9]), is therefore sharper, since it forces $k$ to be even. In the general case $k$ need not be even.

4. Classification of normal subgroups. In this final section we study the normal subgroups between $E(\mathfrak{f})$ and $I(\mathfrak{f}) O(\mathfrak{f})$. The exceptional behavior that occurs when there are more than one modular Jordan components of rank one, and $F$ is not a $p$-adic number field, is also illustrated.

Lemma 4.1. Let $r \in B_{i}^{-1} M_{i}$ and $s \in M_{j}$ where $1 \leqslant i<j \leqslant h$. Then, for any ideal $\mathrm{b}$ and $1 \leqslant k \leqslant h$,

$$
\left[E(r, s), O\left(\mathfrak{b} M_{k}\right)\right] \cong E_{i j}(\mathfrak{b}) .
$$

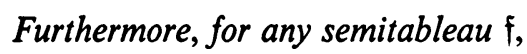

$$
[E(r, s), O(\mathfrak{f})] \leqq E(\mathfrak{f}) .
$$

Proof. The first part is trivial unless $k$ equals $i$ or $j$. Assume $k=i$; the other case is similar. Let $\theta \in O\left(\mathfrak{b} M_{i}\right)$ and $\theta(r)=r+t$ where $t$ is in $\mathfrak{b} \mathfrak{s}_{i}^{-1} M_{i}$. Then

$$
[E(r, s), \theta]=E(r, s) E(r+t,-s) .
$$

We now work in the Clifford algebra. Consider

$$
T=(1+r \otimes s) \otimes(1-(r+t) \otimes s)=\zeta-t \otimes s+q(s) r \otimes t
$$

where $\zeta$ is the unit $1+q(r) q(s)$. Take $\lambda$ in $\mathfrak{\zeta}_{j}$ such that

$$
\lambda(\zeta+q(s) B(r, t))+q(s)=0 .
$$

Then the Clifford element

$$
(1+\lambda r \otimes t) \otimes T=\zeta-\lambda q(r) q(s) q(t)-(t+\lambda q(t) r) \otimes s
$$

induces an isor, try in $E_{i j}(\mathfrak{b})$ since $t+\lambda q(t) r$ is in $\mathfrak{b}_{i}{ }_{i}^{-1} M_{i}$. The inclusion now follows since $1+\lambda r \otimes t$ induces an isometry in the group $O\left(\left(\mathfrak{s}_{j}: \mathfrak{s}_{i}\right) \mathfrak{b} M_{i}\right)$, which is contained in $E_{i j}(\mathfrak{b})$ by Lemma 2.3 .

For the remaining part of the lemma let $\mathfrak{f}=\left\{\mathfrak{a}_{i j}\right\}$ and $\mathfrak{b}_{k}=\mathfrak{a}_{k-1 k} \cap \mathfrak{a}_{k k+1}$. By Theorem 2.7,

$$
O(\mathfrak{f})=E(\mathfrak{f}) O\left(\mathfrak{b}_{1} M_{1}\right) \cdots O\left(\mathfrak{b}_{h} M_{h}\right)
$$

and it suffices to prove $\left[E(r, s), O\left(\mathfrak{b}_{k} M_{k}\right)\right] \leqq E(\mathfrak{f})$ for $1 \leqslant k \leqslant h$. If $k=i$, 


$$
\left[E(r, s), O\left(\mathfrak{b}_{i} M_{i}\right)\right] \leqq E_{i j}\left(\mathfrak{b}_{i}\right) \leqq E_{i j}\left(\mathfrak{a}_{i i+1}\right) \leqq E_{i j}\left(\mathfrak{a}_{i j}\right) \leqq E(\mathfrak{f}) .
$$

The argument for $k=j$ is similar. For other values of $k$ the result is trivial. This proves the lemma.

LEMma 4.2. Let $r \in \mathcal{F}_{i}^{-1} M_{i}$ and $s \in M_{j}$ where $1 \leqslant i<j \leqslant h$. Then, for any semitableau $\mathfrak{f}$,

$$
[E(r, s), I(\mathfrak{f})] \cong E(\mathfrak{f})
$$

Proof. The result is trivial if $I(\mathfrak{f})=\{ \pm I\}$. Assume, therefore, $f$ $=\left\{a_{i j}\right\}$ and $a_{k k+1}=\mathfrak{o}$ for some $k$. It suffices to consider $i \leqslant k<j$, since for other values of $k, \Psi_{k}$ and $E(r, s)$ commute. Now

$$
\left[E(r, s), \Psi_{k}\right]=E(r, s)^{2} \in E_{i j}(0) .
$$

Since $\mathfrak{o}=\mathfrak{a}_{k k+1} \cong \mathfrak{a}_{k j} \cong \mathfrak{a}_{i j} \cong \mathfrak{o}$, we have $E_{i j}(\mathfrak{o}) \subseteq E(\mathfrak{f})$ and the result follows.

LEMMA 4.3. Let $\mathfrak{R}$ be a normal subgroup of $O(M)$ with

$$
E(\mathfrak{f}) \cong \Re \cong I(\mathfrak{f}) O(\mathfrak{f})
$$

for $\mathfrak{f}=\left\{\mathfrak{a}_{i j}\right\}$. If $\varphi=\chi \theta_{1} \cdots \theta_{h} \psi$ is in $\mathfrak{R}$ where $\chi \in I(\mathfrak{f}), \psi \in E(\mathfrak{f})$ and $\theta_{i} \in O\left(\mathfrak{b}_{i} M_{i}\right), 1 \leqslant i \leqslant h$, then $\theta_{k}$ is also in $\mathfrak{R}$, provided $\mathfrak{b}_{k} \cong \mathfrak{p}$.

Proof. Since $\mathfrak{b}_{k} \cong \mathfrak{p}$, it follows that $\operatorname{det} \theta_{k}=1$. If rank $M_{k}=1$, then necessarily $\theta_{k}=I_{k} \in \mathfrak{N}$. Now assume $n=\operatorname{rank} M_{k} \geqslant 2$. Then as in Lemma 3.7,

$$
\theta_{k}=E\left(x_{1}, t_{1}\right) \cdots E\left(x_{n-1}, t_{n-1}\right)
$$

where $x_{1}, \ldots, x_{n}$ is an orthogonal basis for $M_{k}$, and $t_{i}$ $\in \mathfrak{b}_{k} \partial_{k}^{-1} M_{k}$ satisfy $B\left(x_{i}, t_{j}\right)=0$ for $1 \leqslant i \leqslant j \leqslant n-1$. Computing [ $\left[\psi^{-1}{ }^{i}\right.$, $\left.\Psi\left(x_{1}\right)\right] \in \mathfrak{R}$, and observing that $\chi$ and $\Psi\left(x_{1}\right)$ commute, we find that $E\left(x_{1}, t_{1}\right)^{2}$ is in $\mathfrak{R}$. Since $\mathfrak{b}_{k} \cong \mathfrak{p}$, it follows from Lemma 3.1 that $E\left(x_{1}, t_{1}\right)$ is in $\mathfrak{R}$. Similarly, all $E\left(x_{i}, t_{i}\right)$ are in $\mathfrak{N}$. Hence $\theta_{k}$ is in $\mathfrak{R}$.

THEOREM 4.4. Let $\mathfrak{f}=\left\{\mathfrak{a}_{i j}\right\}$ be a semitableau with $\mathfrak{b}_{k}=a_{k-1 k} \cap \mathfrak{a}_{k k+1}$ in $\mathfrak{p}$ for $1 \leqslant k \leqslant h$, and $\mathfrak{N}$ be a subgroup of $O(M)$ satisfying $E(\mathfrak{f}) \cong \mathfrak{N} \cong I(\mathfrak{f}) O(\mathfrak{f})$. Then $\mathfrak{N}$ is normai in $O(M)$ if and only if

$$
\mathfrak{N}=I(\mathfrak{N}) \mathfrak{N}_{1} \cdots \mathfrak{N}_{h} E(\mathfrak{f})
$$

where $I(\mathfrak{N})=\mathfrak{R} \cap I(\mathfrak{f})$ and the $\mathfrak{R}_{k}=\mathfrak{N} \cap O\left(M_{k}\right), 1 \leqslant k \leqslant h$, are normal subgroups of $O\left(M_{k}\right)$ satisfying 


$$
O\left(\mathfrak{c}_{k} M_{k}\right) \cong \mathfrak{N}_{k} \cong O\left(\mathfrak{b}_{k} M_{k}\right)
$$

Proof. That such a subgroup is normal in $O(M)$ follows from Lemmas 4.1 and 4.2 and the fact (Lemma 3.2) that $O(M)$ is generated by the $E(r, s)$ in these two lemmas, together with the subgroups $O\left(M_{k}\right), 1 \leqslant k \leqslant h$. Conversely, if $\mathfrak{R}$ is normal in $O(M)$, the given decomposition follows from Lemma 4.3 and Theorem 2.10.

REMARK 4.5. Theorem 4.4 completes the classification of normal subgroups associated with a semitableau $\mathfrak{f}$ having all $\mathfrak{b}_{k} \cong \mathfrak{p}$, since the possible $\mathfrak{N}_{k}$ are easily determined. Thus

(i) If rank $M_{k}=1$, necessarily $\mathfrak{N}_{k}=\left\{I_{k}\right\}$.

(ii) If rank $M_{k}=2$, all subgroups of $O^{+}\left(M_{k}\right)$ between $O\left(\mathfrak{c}_{k} M_{k}\right)$ and $O\left(\mathfrak{b}_{k} M_{k}\right)$ are normal in $O\left(M_{k}\right)$. The structure of the 2-dimensional group $O^{+}\left(M_{k}\right)$ is well known.

(iii) If rank $M_{k} \geqslant 3$, then $\mathfrak{N}_{k}$ is a congruence subgroup in $O\left(M_{k}\right)$. Thus $\mathfrak{R}_{k}=O\left(\mathfrak{d}_{k} M_{k}\right)$ where $\mathfrak{c}_{k} \subseteq \mathfrak{d}_{k} \cong \mathfrak{b}_{k}$. This is well known, e.g. [5]; it also follows from (f) in $\S 3$, using the decomposition of $\theta_{k} \in \mathfrak{N}_{k}$ as in Lemma 4.3.

If rank $M_{k} \geqslant 3$ for all $k=1, \ldots, h$, then (iii) above leads quickly to the classification given by Riehm [10], using Remark 2.12, although in [10] the more general problem of classifying subgroups normalized by the commutator subgroup of $O(M)$ is considered.

With a normal subgroup $\mathfrak{R}$ satisfying $E(\mathfrak{f}) \subseteq \mathfrak{N} \cong I(\mathfrak{f}) O(\mathfrak{f})$ for a semitableau $\mathfrak{f}$ having some $\mathfrak{b}_{k}=\mathfrak{d}$, a splitting of $\mathfrak{N}$ into a product of subgroups as in Theorem 4.4 is not always possible, although all such split subgroups are normal in $O(M)$. This splitting is not possible for $O^{+}(M)$ or the spinorial kernel of $O(M)$. A detailed classification of normal subgroups for $f$ with some $\mathfrak{b}_{k}$ 's equal to $o$ would be complex and we do not pursue it here (see also [10, Theorem 7.7] for a special case).

EXAMPLE 4.6. The following example illustrates the type of exceptional behavior that occurs if there are more than one Jordan components with rank one. Let $\mathfrak{o}=\mathbf{F}[[X]]$ be the power series ring over a finite field $\mathbf{F}$ with characteristic $p$ and

$$
M=\mathfrak{o x} \perp \text { oy } \perp L
$$

where $q(x)=1, q(y) 0=s_{2} \varsubsetneqq s_{1}=0$ and $L$ is a modular lattice with scale $\mathfrak{s}_{3} \subsetneq \mathfrak{s}_{2}$. For an ideal $\mathfrak{a}=\alpha \mathfrak{d}$, take $\mathfrak{f}=\left\{a_{i j}\right\}$ to be the semitableau

$$
\mathfrak{a}_{12}=\left(\mathfrak{g}_{3}: \mathfrak{g}_{2}\right) \mathfrak{a}, \quad \mathfrak{a}_{13}=\mathfrak{a}, \quad \mathfrak{a}_{23}=\mathfrak{z}_{2} \mathfrak{a}
$$

and $\mathfrak{R}$ to be the subgroup in $O(M)$ generated by $E(\mathfrak{f})$ together with the isometry $E(x, \alpha y)$. We show below that $\mathfrak{N}$ is a normal subgroup. Also, since 
$E(x, \alpha y)^{p}=I$, it will follow that $[\Re: E(\mathfrak{f})]=p$. It is then clear that, in general, $E(\mathfrak{f})$ is the largest subgroup of this type in $\mathfrak{R}$. However, $\mathfrak{N}$ is not contained in $I(\mathfrak{f}) O(\mathfrak{f})$, and consequently the main theorem from the last section fails to hold in this case.

By Lemma 3.2, $O(M)$ is generated by $\Psi(x), \Psi(y), O(L)$, the isometries $E(x, \beta y)$ with $\beta$ in 0 , and the isometries $E(x, z)$ and $E\left(q(y)^{-1} y, z\right)$ with $z$ in $L$. To prove $\mathfrak{N}$ is a normal subgroup, it suffices therefore, to show both $[E(x, \alpha y), E(x, z)]$ and $\left[E(x, \alpha y), E\left(q(y)^{-1} y, z\right)\right]$ lie in $E_{13}(a) E_{23}\left(\xi_{2} a\right)$, and hence in $E(\mathfrak{f})$, for all $z$ in $L$. We indicate a proof for the first of these; the second is similar. Here

$$
[E(x, \alpha y), E(x, z)]=E(x, \alpha y) E(u,-\alpha y)
$$

where

$$
u=E(x, z)(x)=x-2(1+q(z))^{-1}(q(z) x+z) .
$$

By expanding $(1+x \otimes \alpha y) \otimes(1-u \otimes \alpha y)$ in the Clifford algebra as in Lemma 4.1 , it is easily seen that $E(x, \alpha y) E(u,-\alpha y)$ is now in $E_{23}\left(\mathfrak{g}_{2} \mathfrak{a}\right) E_{13}\left(\mathfrak{g}_{2} \mathfrak{a}^{2}\right)$, and the result follows.

Variations of this type of exceptional behavior will also occur when the valuation ring $o$ has characteristic zero if either $p$ ramifies or the residue class field is not a prime field.

\section{REFERENCES}

1. L. Bröcker, Orthogonale Gruppen über diskret bewerteten vollständigen Körpern, Abh. Math. Sem. Univ. Hamburg 34 (1969/70), 238-251. MR 42 \#3188.

2. D. G. James, On the structure of orthogonal groups over local rings, Amer. J. Math. 95 (1973), 255-265. MR 48 \#8653.

3. - Orthogonal groups of three dimensional anisotropic quadratic forms, J. Algebra 37 (1975), 121-136.

4. R. P. Johnson, Orthogonal groups of local anisotropic spaces, Amer. J. Math. 91 (1969), 1077-1105. MR 41 \# 1893.

5. W. Klingenberg, Orthogonale Gruppen über lokalen Ringen, Amer. J. Math. 83 (1961), 281-320. MR 23 \#A1726.

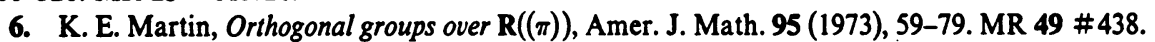

7. O. T. O'Meara, Introduction to quadratic forms, Grundlehren der math. Wiss., Band 117, Springer-Verlag, Berlin and New York, 1963. MR 27 \#2485.

8. B. Pollak, On the structure of local orthogonal groups, Amer. J. Math. 88 (1966), 763-780. MR 34 \#4369.

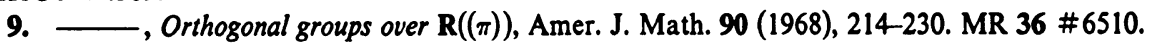

10. C. R. Riehm, Orthogonal groups over the integers of a local field. II, Amer. J. Math. 89 (1967), 549-577. MR 35 \#6765.

11. - The congruence subgroup problem over local fields, Amer. J. Math. 92 (1970), 771-778. MR 42 \#3090.

Department of Ma'irmatics, Pennsylvania State University, University Park, PennsylVANIA 16802 